Okajimas Folia Anat. Jpn., 56(4) : 193-204, October 1979

\title{
Localization of Concanavalin A Binding Sites with a Concanavalin A-Alkaline Phosphatase Method
}

\author{
By \\ SATORU SHIMIZU and KAZUYori YAMADA* \\ Laboratory of Ultrastructure Research, Aichi Cancer Center Research Institute, \\ Nagoya 464 and $\left(^{*}\right)$ Laboratory of Histology, Department of Anatomy, \\ Nagoya University School of Medicine, Nagoya 466, Japan \\ -Received for Publication, February 1, 1979-
}

\begin{abstract}
Key Words: Histochemical method, Complex carbohydrates, Concanavalin A, Alkaline phosphatase.

Summary. In a series of different vertebrate tissues, concanavalin A binding sites were localized precisely by an alkaline phosphatase-labelled concanavalin A-diazo blue B (Con A-ALP-DBB) method. The results obtained with control staining procedures for the Con A-ALP-DBB method substantiated the usefulness of this staining method. The successful use of Con A-ALP conjugate in the Con A-ALP-DBB staining method is regarded as a starting point for the development of Con A-ALP methods which are better than Con A-peroxidase (PO) methods in the preservation of tissue sections and are applicable to tissue specimens for both light and electron microscopy.
\end{abstract}

\section{Introduction}

Bernhard and Avrameas (1971) developed a concanavalin A-peroxidase-diaminobenzidine (Con A-PO-DAB) method for the histochemical demonstration of $\alpha$-Dglucosyl and $\alpha$-D-mannosyl residues of complex carbohydrates on cell surfaces by electron microscopy. Attempts have subsequently been made to utilize this method for the detection of particular saccharide residues not only in electron microscopy (Parmley, Martin and Spicer, 1973; Guillouzo and Feldmann, 1977) but also in light microscopy (Kiernan, 1975; Yamada and Shimizu, 1976; Yamada, 1978). In electron microscopy, however, the demonstration of particular saccharide residues of complex carbohydrates involved in the intracellular structures has hardly been possible, inasmuch as the reagents employed such as Con $\mathrm{A}$ and $\mathrm{PO}$ show only limited penetration into cells and tissues. In order to overcome such difficulties, Bretton and Bariety (1976) and Gros, Obrénovitch, Challice, Monsigny and Schrével (1977) applied the Con A-PO-DAB method to glycol methacrylate (GMA) embedded ultrathin sections of various organs of mammals and obtained images of the ultrastructural histochemistry which were satisfactory in guality. However, close examination of their images reveals that the tissue sections were apparently injured due to the use of hydrogen peroxide in the DAB reaction. Employment of a better staining method with the same staining specificity which is free from the disadvantage of injuring the 
tissue sections, is thus needed. As a starting point for the development of such a staining method in electron microscopy, an alkaline phosphatase-labelled concanavalin A (Con A-ALP) method was tested in the present study in a series of vertebrate tissue specimens for light microscopy. The results obtained indicate that the Con A-ALP method represents a promising staining procedure for studies on complex carbohydrates with particular saccharide residues in light and electron microscopic histochemistry.

\section{Materials and Methods}

Tissue specimens containing large amounts of complex carbohydrates were obtained from different vertebrate species. These included umbilical cord (man, pig, cattle), tracheal cartilage (rat, rabbit), ear cartilage (rat), costal cartilage (rabbit), gill cartilage (eel), aorta (dog, pig, cattle, rabbit), comb (cock), stomach (rat, rabbit), jejunum (rat), colon (rabbit) and sublingual gland (rat, mouse). The tissue blocks obtained from each donor were cut into cubes with a side of approximately 5 to $10 \mathrm{~mm}$. All tissue pieces were fixed in chilled $\left(4^{\circ} \mathrm{C}\right) 10 \%$ formalin buffered with phosphate (Lillie and Fullmer, 1976), in chilled $\left(4^{\circ} \mathrm{C}\right) 2 \%$ calcium acetate in $10 \%$ formalin (Leppi, 1968) or in chilled $\left(4^{\circ} \mathrm{C}\right) \quad 10 \%$ formalin in $95 \%$ ethanol (McManus and Mowry, 1958) for 12 to $24 \mathrm{hr}$. After fixation, the tissue pieces were dehydrated in a graded ethanol series of ascending concentrations and embedded in paraffin, according to the usual methods. Sections with a thickness of 6 to $9 \mu$ were cut on a sliding microtome, affixed on slides, deparaffinized with xylene, hydrated through an ethanol series of descending concentrations and subjected to the following staining procedures for the light micro- scopic localization of Con A binding sites in complex carbohydrate-containing tissues.

\section{Staining Procedures}

[A] The alkaline phosphatase-labelled concanavalin A-diazo blue B (Con AALP-DBB) procedure

Alkaline phosphatase was prepared from $E$. coli B by the method of Malamy and Horecker (1964). Concanavalin A was purchased from Miles-Yeda. Conjugation of the lectin $(200 \mu \mathrm{g} / \mathrm{ml})$ with the enzyme $(100 \mu \mathrm{g} / \mathrm{ml})$ was performed in $0.2 \% \mathrm{glu}$ taraldehyde at $37^{\circ} \mathrm{C}$ and, after $2.5 \mathrm{hr}$, ethanolamine ( $\mathrm{pH} 9.0$ ) was added to the reaction mixture at a final concentration of $0.1 \mathrm{M}$. The conjugate was purified by chromatography on Sephadex G-200, while washing with phosphate buffered saline (PBS), and elution with $0.1 \mathrm{M} \alpha$ methyl-D-mannoside was followed by monitoring both the ultraviolet light absorption and alkaline phosphatase activity. The purified preparation so obtained was dialyzed overnight against a large volume of PBS. It was employed as a working solution of Con A-ALP in the Con A-ALP-DBB staining procedure. This procedure was performed according to the following sequence.

The hydrated sections were .

(1) rinsed in distilled water for $5 \mathrm{~min}$;

(2) rinsed in phosphate buffered saline (PBS) $[0.05 \mathrm{M}$ phosphate buffer $(\mathrm{pH}$ 7.2) containing $0.2-0.5 \mathrm{M} \mathrm{NaCl}]$ for $5 \mathrm{~min}$;

(3) incubated in the working solution of Con A-ALP conjugate for 15 to 30 min at room temperature;

(4) rinsed in PBS for $5 \mathrm{~min}$;

(5) incubated for 20 to $40 \mathrm{~min}$ at $37^{\circ} \mathrm{C}$ in a medium for the alkaline phosphatase reaction $[50 \mathrm{mg}$ of naphthol AS-BI phosphate was dissolved in $0.25 \mathrm{ml}$ of dimethyl sulfoxide, to 
which $25 \mathrm{ml}$ of distilled water and $25 \mathrm{ml}$ of $0.2 \mathrm{M}$ Tris- $\mathrm{HCl}$ buffer $(\mathrm{pH}$ 8.3) were added. Then, $30 \mathrm{mg}$ of Fast blue B (diazo blue B) was added, and the solution mixed well and filtered, before use] [modification of Burstone's (1961) method];

(6) washed in distilled water for 5 to $10 \mathrm{~min}$;

(7) mounted in glycerine or glycerinejelly.

\section{[B] Control staining procedures}

(a) Con A-ALP with $\alpha$-methyl-D-mannoside-DBB procedure

In order to confirm that the Con AALP conjugate exhibits specific binding affinity towards particular saccharide residues of complex carbohydrates, a Con A-ALP with $\alpha$-methyl-D-mannosideDBB procedure was carried out as a control staining procedure. At step (3) of the above sequence, some control tissue sections were incubated in Con A-ALP solution containing $50 \mathrm{mg} / \mathrm{ml}$ of $\alpha$-methyl-D-mannoside for 15 to $30 \mathrm{~min}$ at room temperature. Following this, the sections were treated sequentially, according to steps (4) to (7) of the original staining procedure.

(b) Con A-ALP-DBB (without enzyme substrate) procedure

As a control staining procedure for detection of the alkaline phosphatase activity of Con A-ALP, a Con A-ALP-DBB (without enzyme substrate) procedure was performed. At step (5) of the above sequence, some other control tissue sections were incubated for 20 to $40 \mathrm{~min}$ at $37^{\circ} \mathrm{C}$ in a medium for the alkaline phosphatase reaction without naphthol AS-BI phosphate, and then subjected to steps (6) and (7) of the original staining procedure.

(c) DBB (with enzyme substrate) procedure
In order to detect the activity of endogenous alkaline phosphatase in the tissues, still other control tissue sections were processed according to steps (4) to (7) of the Con A-ALP-DBB procedure, omitting steps (1) to (3). Thus, the sections were reacted for DBB (with enzyme substrate) only.

(d) The Con A-PO-DAB procedure

For the purpose of confirming that the Con A-ALP-DBB procedure gives staining patterns identical to those obtained by the Con A-PO-DAB procedure, certain other control tissue sections were subjected to a Con A-PO-DAB procedure reported previously (Yamada and Shimi$\mathrm{zu}, 1976)$. The staining images obtained with both types of procedures were compared for all the different tissue specimens tested.

\section{Results}

[A] The Con A-ALP-DBB procedure

In the majority of tissues employed, the Con A-ALP-DBB procedure colored stainable histologic structures a purple or blue shade of varying intensity.

In human, porcine and bovine umbilical cord, the stroma and arterial stroma exhibited a positive Con A-ALP-DBB reaction which was moderate or relatively strong in intensity (Figs. 1 and 2). In the tracheal cartilage of the rat and rabbit, ear cartilage of the rat, costal cartilage of the rabbit and gill cartilage of the eel, the Con A-ALP-DBB reaction of the intercellular matrix was feeble or moderate in intensity, whereas that of the lacunar border and chondrocyte cytoplasm was moderate or strong in intensity (Fig. 3). In the aorta of the dog, pig, cattle and rabbit, the subendothelial spaces of the intima and interelastic spaces of the media showed a positive Con A-ALP-DBB reaction which was variable in intensity at different 
loci of the tissues and was moderate or strong in intensity (Eig. 4). In the dermoid layer of cockscomb, the fibers exhibited a moderately or strongly positive Con A-ALP-DBB reaction, while the reaction of the stroma was either weak or moderate in intensity (Fig. 5). In the stomach of the rat and rabbit, both the surface and foveolar mucus showed a positive Con A-ALP-DBB reaction which was, however, feeble or moderate in intensity. In the jejunum of the rat, the Con A-ALP-DBB reaction of the goblet cells was either doubtful or faint in intensity, but the columnar cells and subjacent connective tissue elements showed a moderately or strongly positive reaction (Fig. 6). In the colon of the rabbit, the superficial goblet cells and goblet cells upper and deep in the crypt exhibited a positive Con AALP-DBB reaction which was weak or moderate in intensity (Fig. 7). In the sublingual gland of the rat and mouse, the Con A-ALP-DBB reaction of the acini, ductuli and ducts was moderate or strong in intensity (Fig. 8).

\section{[B] Control staining procedures}

Addition of $\alpha$-methyl-D-mannoside to the Con A-ALP solution employed for the Con A-ALP.DBB procedure abolished the positive Con A-ALP-DBB reaction in the majority of the histologic structures examined (Figs. 9, 10 and 11). In a few histologic structures such as the intercellular matrix of rabbit costal cartilage and dermaid fibers of cockscomb, however, a residual but faintly positive Con A-ALP-DBB reaction was noted, even after the addition of $\alpha$-methyl-D-mannoside.

When tissue sections were subjected to the Con A-ALP-DBB (without enzyme substrate) staining procedure, all the histologic structures tested failed to exhibit a positive Con A-ALP-DBB reaction
(Figs. 12 and 13). Such negative Con A-ALP-DBB reaction was observed in all tissues examined without exception.

In control tissue sections subjected to the DBB (with enzyme substrate) procedure for demonstrating the activity of endogenous alkaline phosphatase, the majority of the histologic structures tested were not stained positively at all: However, a few histologic structures such as the free surfaces of columnar cells of the jejunum of the rat and myoepithelial cell cytoplasm in the sublingual gland of the rat and mouse, were colored a purple or blue shade of detectable intensity (Fig. 14).

In the majority of histologic structures examined in the present study, the Con A-PO-DAB staining procedure resulted in staining patterns which were almost identical in intensity and distribution to those obtained by the Con A-ALP-DBB procedure (Figs. 15 and 16). The only difference between the two sets of staining patterns was that the Con A-PO-DAB reaction tended to be more vivid as regards intensity than the Con A-ALPDBB reaction in a few histologic structures such as the stroma and arterial stroma of human umbilical cord, the lacunar border of rabbit costal cartilage and the dermoid fibers of cockscomb.

\section{Discussion}

The present study was undertaken as a starting point for the development of a method of detecting Con A binding sites other than Con A-PO-DAB, in which irritative reagents such as hydrogen peroxide were not used so as to ensure better preservation of the tissue sections, in particular ultrathin sections for electron microscopy. As a result, a Con AALP-DBB method was successfully applied to tissue sections from a series of vertebrate organs for light microscopy. 
The results obtained with the Con A-ALP with $\alpha$-methyl-D-mannoside-DBB procedure are taken to indicate that the Con A-ALP conjugate employed exhibits a specific binding affinity towards particular saccharide residues of complex carbohydrates such as $\alpha$-D-mannosyl residues (Kiernan, 1975; Yamada and Shimizu, 1976). In addition, the Con A-ALPDBB (without enzyme substrate) procedure yielded results which confirmed that the enzyme activity of the Con A-ALP conjugate is specifically demonstrated by the ensuing DBB step of the staining procedure.

The specific binding affinity of the Con A-ALP conjugate towards particular saccharide residues was, furthermore, substantiated by the finding that the staining patterns obtained with the Con A-ALP-DBB procedure were almost identical in intensity and distribution to those obtained by the Con A-PO-DAB procedure.

All the above results appear to confirm that Con A-ALP methods such as that employed in the present study represent useful staining procedures permitting the histochemical detection of Con A binding sites without noticeable injury to the tissue sections for light and electron microscopy. When the Con A-ALP methods are to be applied to ultrathin sections for electron microscopy, the alkaline phosphatase activity of Con A-ALP conjugate may be visualized by heavy metal precipitation techniques such as the lead nitrate (Hugon and Borgers, 1966) and lead citrate (Mayahara, Hirano, Saito and Ogawa, 1967) methods.

As in the case of the Con A-PO-DAB (Kiernan, 1975; Yamada and Shimizu, 1976; Yamada, 1978) and Ricinus communis agglutinin (RCA)-PO-DAB (Yamada and Shimizu, 1977) staining procedures, the activity of endogenous enzyme (alkaline phosphatase) gives rise to confusion in the interpretation of the results obtained with the Con A-ALP-DBB staining procedure. Such enzyme activity may be checked by a DBB (with enzyme substrate) procedure performed on control tissue sections. Except for this inconvenience, the Con A-ALP methods such as that described here are thought to provide a promising means of localizing Con A binding sites precisely in light and electron microscopy.

\section{Acknowledgements}

The authors wish to express their sincere thanks to Dr. K. Kimata, Faculty of Science, Nagoya University, for his kind assistance in purifying alkaline phosphatase of Escherichia coli.

\section{References}

1) Bernhard, W. and Avrameas, S. (1971) Ultrastructural visualization of cellular carbohydrate components by means of concanavalin A. Exp. Cell Res., 64: 232-236.

2) Bretton, R. and Bariety, J. (1976) A comparative ultrastructural localization of concanavalin A, wheat germ and Ricinus communis on glomeruli of normal rat kidney. J. Histochem. Cytochem., 24 : 1093-1100.

3) Burstone, M.S. (1961) Histochemical demonstration of phosphatases in frozen sections with naphthol AS phosphates. J. Histochem. Cytochem., 9 : 146-153.

4) Gros, D., Obrénovitch, A., Challice, C.E., Monsigny, M. and Schrével, J. (1977) Ultrastructural visualization of cellular carbohydrate components by means of lectins on ultrathin glycol methacrylate sections. J. Histochem. Cytochem., 25 : 104-114.

5) Guillouzo, A. and Feldmann, G. (1977) Surface and intracellular localization of concanavalin A binding sites in rat liver cells. J. Histochem. Cytochem., $25: 1303-$ 1310. 
6) Hugon, J. and Borgers, M. (1966) A direct lead method for the electron microscopic visualization of alkaline phosphatase activity. J. Histochem. Cytochem., $14:$ 429-431.

7) Kiernan, J.A. (1975) Localization of $\alpha$ D-glucosyl and $\alpha$-D-mannosyl groups of mucosubstances with concanavalin A and horseradish peroxidase. Histochemistry $44: 39-45$.

8) Leppi, T.J. (1968) Morphochemical analysis of mucous cells in the skin and slime glands of hagfishes. Histochemie 15: 68-78.

9) Lillie, R.D. and Fullmer, H.M. (1976) Fixation. In: Histopathologic Technic and Practical Histochemistry (4th Edn), New York, McGraw-Hill, 25-68.

10) Malamy, M.H. and Horecker, B.L. (1964) Purification and crystalization of the alkaline phosphatase of Escherichia coli. Biochemistry 3 : 1893-1897.

11) Mayahara, H., Hirano, H., Saito, T. and Ogawa, K. (1967) The new lead citrate method for the ultracytochemical demonstration of non-specific alkaline phosphatase (orthophosphoric monoester phos- phohydrolase). Histochemie $11: 88-96$.

12) McManus, J.F.A. and Mowry, R.W. (1958) Effects of fixation on carbohydrate histochemistry. J. Histochem. Cytochem., 6 : 309-316.

13) Parmley, R.R., Martin, B.J. and Spicer, S.S. (1973) Staining of blood cell surfaces with a lectin-horseradish peroxidase method. J. Histochem. Cytochem., 21 : 912-922.

14) Yamada, K. and Shimizu, S. (1976) Concanavalin A-peroxidase-diaminobenzidine (Con A-PO-DAB)-alcian blue (AB). A reliable method for dual staining of complex carbohydrates. Histochemistry 47 : 159-169.

15) Yamada, K. and Shimizu, S. (1977) The histochemistry of galactose residues of complex carbohydrates as studied by peroxidase-labeled Ricinus communis ag. glutinin. Histochemistry 53 : 143-156.

16) Yamada, K. (1978) Concanavalin A-peroxidase-diaminobenzidine-periodic acidm-aminophenol-Fast Black salt $\mathrm{K}$ : a method for the dual staining of neutral complex carbohydrates. Histochemical J., 10 : 573-584. 


\section{PLATES}




\section{Explanation of Figures}

\section{Plate I}

Fig. 1. Human umbilical cord stained with Con A-ALP-DBB. The stroma and arterial stroma reacted moderately or strongly. $\times 68$.

Fig. 2. Porcine umbilical cord stained with Con A-ALP-DBB. The stroma and arterial stroma reacted moderately or strongly. $\times 68$.

Fig. 3. Rat ear cartilage stained with Con A-ALP-DBB. The intercellular matrix reacted feebly or moderately, whereas the lacunar border and chondrocytes were stained moderately or vividly. $\times 180$.

Fig. 4. Bovine aorta stained with Con A-ALP-DBB. The subendothelial spaces of the intima and interelastic spaces of the media exhibited a positive reaction of variable intensity. $\times 68$.

Fig. 5. Cockscomb stained with Con A-ALP-DBB. The reaction of the dermoid fibers was moderate or strong in intensity, while the stroma reacted weakly or moderately. $\times 68$.

Fig. 6. Rat jejunum stained with Con A-ALP-DBB. The goblet cells reacted doubtfully or faintly, whereas the columnur cells and subjacent connective tissue elements showed a moderately or strongly positive reaction. $\times 170$.

Fig. 7. Rabbit colon stained with Con A-ALP-DBB. The goblet cells reacted variably. $\times 170$.

Fig. 8. Mouse sublingual gland stained with Con A-ALP-DBB. The acini, ductuli and ducts exhibited a moderately or strongly positive reaction. $\times 170$. 

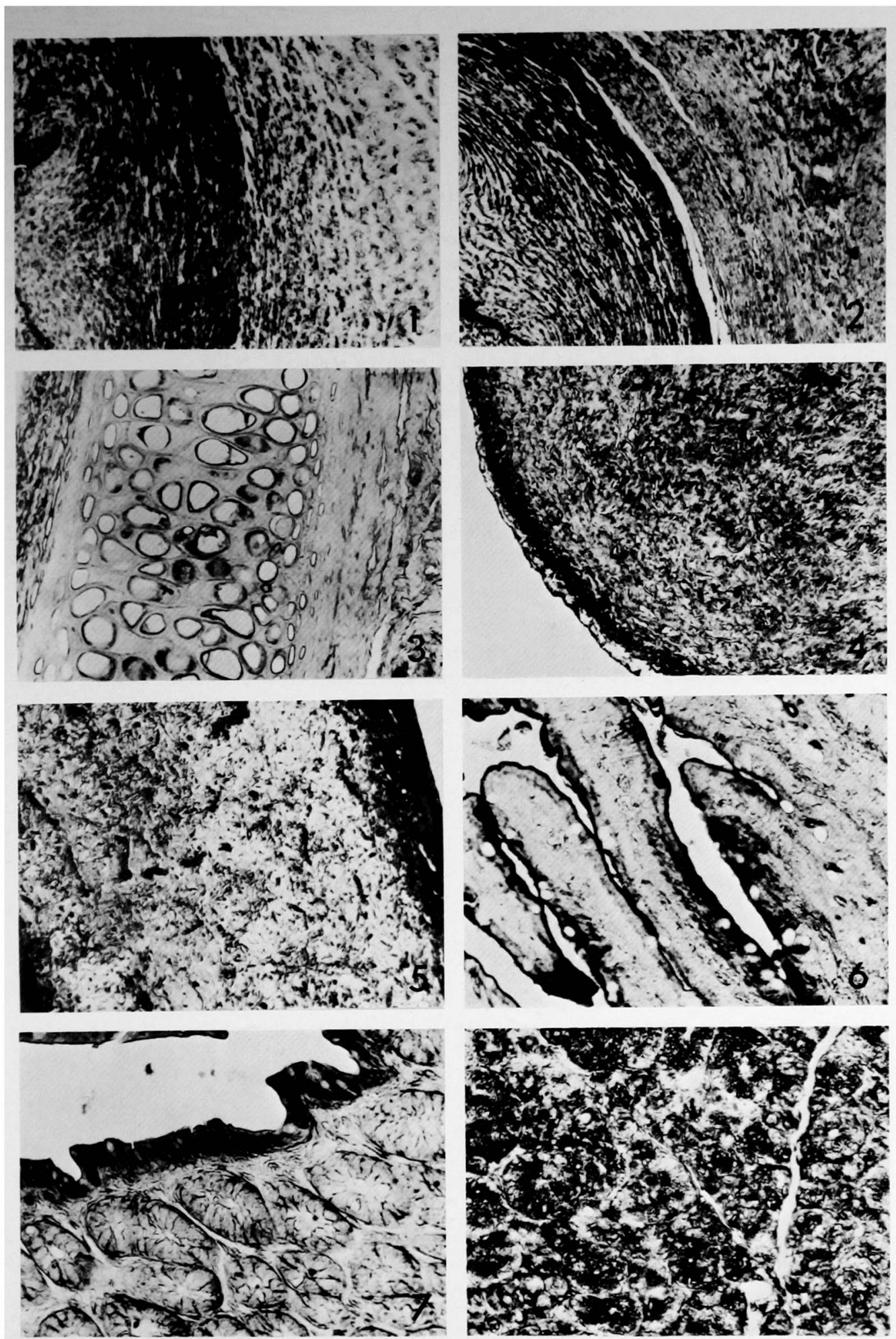


\section{Plate II}

Fig. 9. Porcine umbilical cord stained with Con A-ALP with $\alpha$-methyl-D-mannoside-DBB. The reaction of the stroma and arterial stroma was negative or faint. $\times 68$.

Fig. 10. Cockscomb stained with Con A-ALP with $\alpha$-methyl-D-mannoside-DBB. The dermoid flbers and stroma exhibited a negative or feeble reaction. $\times 68$.

Fig. 11. Mouse sublingual gland stained with Con A-ALP with $\alpha$-methyl-D-mannoside-DBB. The acini, ductuli and ducts failed to exhibit a positive reaction. $\times 170$.

Fig. 12. Bovine aorta stained with Con A-ALP-DBB (without enzyme substrate). The subendothelial spaces of the intima and interelastic spaces of the media exhibited a negative reaction. $\times 68$.

Fig. 13. Rat jejunum stained with Con A-ALP-DBB (without enzyme substrate). The goblet cells, free surfaces of the columnar cells and subjacent connective tissue elements exhibited a negative reaction. $\times 170$.

Fig. 14. Rat jejunum stained only with DBB (with enzyme substrate). The free surfaces of the columnar cells showed a positive reaction due to endogenous alkaline phosphatase activity. $\times 170$.

Fig. 15. Porcine umbilical cord stained with Con A-PO-DAB. The stroma and arterial stroma reacted moderately or strongly. $\times 68$.

Fig. 16. Cockscomb stained with Con A-PO-DAB. The reaction of the dermoid fibers was strong in intensity, whereas the stroma reacted weakly or moderately. $\times 68$. 


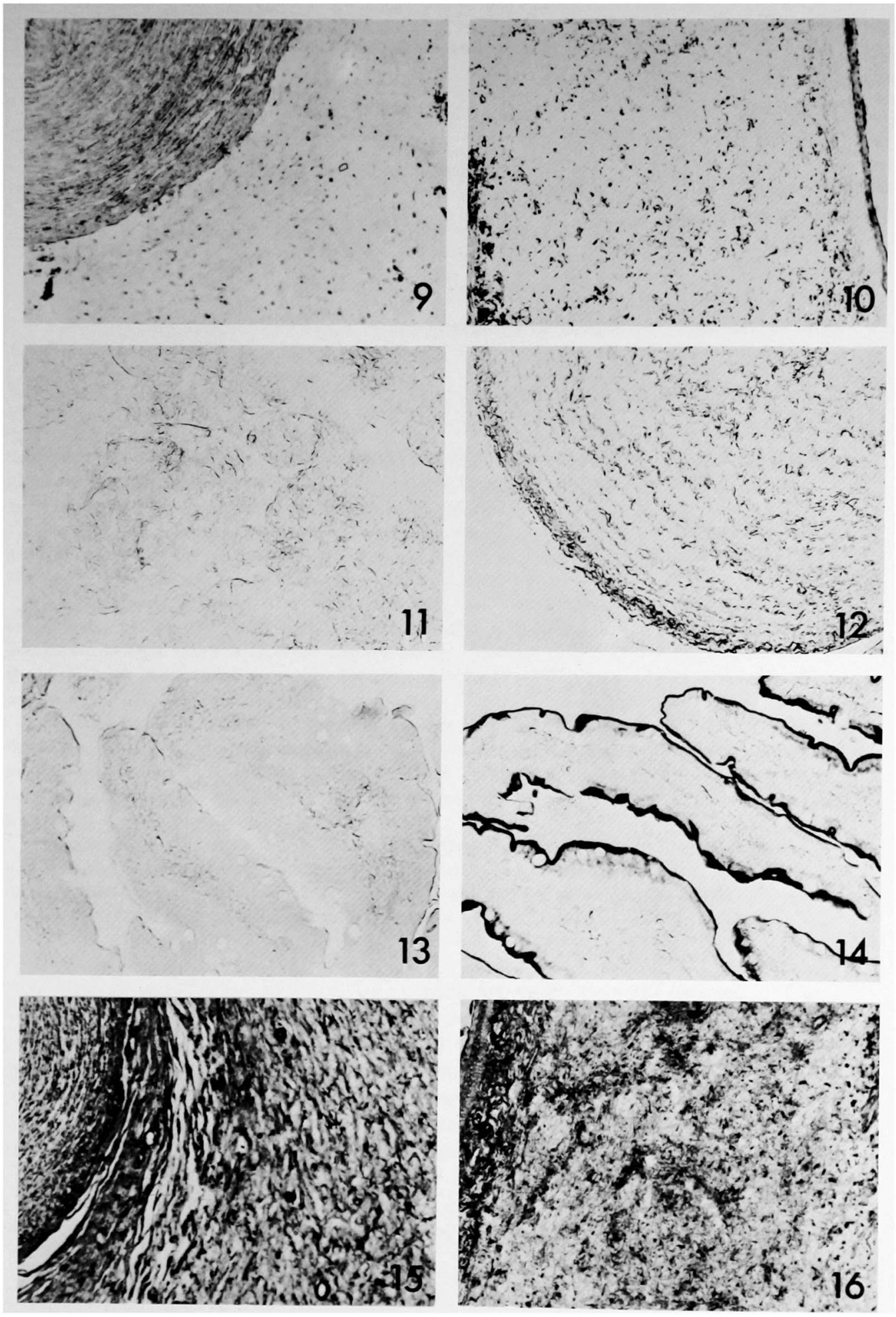

S. Shimizu and K. Yamada 